\title{
Early Diagnosis of Congenital Heart Disease Improves Outcome
}

\author{
Mehnaz Atiq ${ }^{1 *}$ \\ ${ }^{1}$ Section Pediatric Cardiology, Department of Pediatrics, Liaquat National Hospital and Medical College, Karachi, Pakistan
}

\begin{abstract}
Congenital heart disease has an overall incidence of 8-10/1000 live births and is similar across the globe. The incidence may be higher in countries where consanguinity is high, suggesting an autosomal recessive gene as a risk factor. Advances in surgical expertise, have improved outcome of simple and complex heart diseases. Early diagnosis and referral to centers caring for such babies is an important contributory factor to a better outcome.

This review focuses on early diagnosis of congenital heart disease in neonates and children by Health Care Physicians (General and Family Physicians) and Pediatricians. Careful neonatal and pediatric cardiovascular examination, screening pulse oximetry on all newborns before hospital discharge and an early post natal follow up is important to diagnose CHD.
\end{abstract}

Keywords: Congenital heart disease, pulse oximetry screening, cardiac surgery.

\section{INTRODUCTION}

Congenital heart disease ( $\mathrm{CHD}$ ) is one of the most serious birth defects occurring in 8-10/1000 live births and is responsible for $3 \%$ of all infant deaths [1]. Forty-six percent of deaths are due to congenital malformations and congenital heart disease. The actual incidence of CHD may be much higher if still-born fetuses and abortuses are accounted for [2], in whom the incidence appears to be approximately 5 times higher than in live born infants [3]. If not diagnosed early these defects lead to significant morbidity and life threatening events [4].

Given the incidence of CHD and a birth rate of $2.16 \%$ and a population of 208 million (Pakistan demographics' profile, 2019) [5], around 65,000 babies are born with congenital heart disease every year in Pakistan. Around $5 \%$ of these will present as a neonatal cardiovascular emergency.

Advances in medical and surgical management of $\mathrm{CHD}$ have resulted in improved outcome in diseases which previously carried high mortality. Pivotal to such outcomes is early diagnosis and timely referral to centers offering this expertise [6].

\section{ETIOLOGY AND RISK FACTORS FOR CHD}

The etiology of $\mathrm{CHD}$ is hypothesized to be multifactorial, which includes factors like genetic predisposition, environmental insults and several unidentified factors.

Risk factors for the development of $\mathrm{CHD}$ include maternal co-morbidities like hypertension, diabetes mellitus, infections like rubella, use of anti-epileptic drugs, alcohol, antibiotics during pregnancy $[3,7]$, oral contraceptives, exposure to radiation and assisted

\footnotetext{
*Corresponding Author: Mehnaz Atiq, Department of Pediatrics, Liaquat National Hospital and Medical College, Karachi, Pakistan;

Email:mehnaz.atiq@lnh.edu.pk

Received: April 13, 2020; Revised: May 02, 2020; Accepted: May 11, 2020
}

DOI: https://doi.org/10.37184/lnjpc.2707-3521.1.21 reproductive technology. Family history of $\mathrm{CHD}$ is an independent risk factor, particularly if one of the parents has CHD (20-25\% recurrence in offspring). Children with syndromes are also at a higher risk of having $\mathrm{CHD}$ as are those with associations, namely VACTERL (vertebral defects, anal atresia, cardiac defects, tracheoesophageal fistula, renal anomalies and limb abnormalities) and CHARGE (coloboma, heart defects, atresia choanae, growth retardation, genital abnormalities, and ear abnormalities).

The incidence on CHD may be higher in inbred populations like Pakistan [3, 7-10]. Consanguinity has been identified as a risk factor in the Middle Eastern countries with an increase in incidence by 1.8 times in consanguineous parents as compared to unrelated parents [8]. One study from India was unable to identify autosomal recessive inheritance in a heterogeneous cohort of children with CHD [9].

\section{TYPES OF CONGENITAL HEART DISEASES}

CHD are categorized as acyanotic CHDs (with shunts and with obstructions) and cyanotic CHDs. Table 1 lists these defects. The most common acyanotic CHD is the ventricular septal defect, which has an incidence of 4/1000 live births, followed by atrial septal defect with an incidence of $2 / 1000$ live births [1]. Among cyanotic $\mathrm{CHD}$, tetralogy of Fallot is the most common, having an incidence of $0.5 / 1000$ live births.

Table 1: Categories of congenital heart defects.

\begin{tabular}{l} 
Acyanotic CHD \\
With shunt \\
Ventricular septal defect including double outlet right ventricle \\
Atrial septal defect \\
Patent ductus arteriosus \\
Atrioventricular septal defect \\
Obstructive \\
Aortic stenosis \\
Pulmonary stenosis \\
Coarctation of aorta \\
Interrupted aortic arch \\
\hline
\end{tabular}




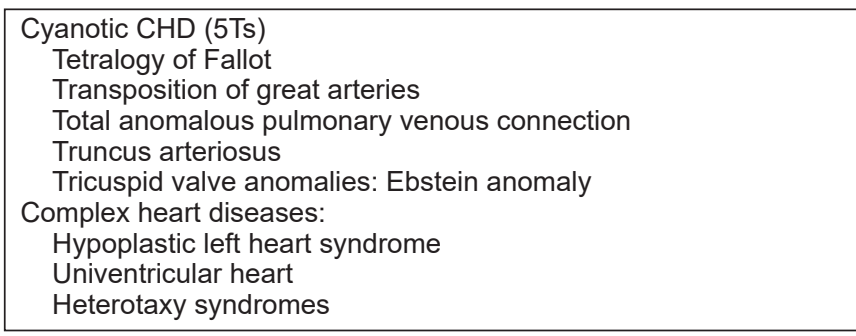

\section{CRITICAL CONGENITAL HEART DISEASE (CCHD)}

CCHD is defined by the Task Force on Screening Critical Congenital Heart Disease [11] as congenital heart diseases which are duct dependent or may require surgical or catheter intervention in the first month of life. CCHDs constitute $25 \%$ of all CHDs [1]. Common defects classified as CCHD are listed in Table 2.

Table 2: Critical congenital heart diseases.

\begin{tabular}{|l|}
\hline d-Transposition of great arteries \\
Pulmonary atresia with or without septal defect \\
Critical pulmonary stenosis with or without septal defect \\
Tricuspid atresia with critical pulmonary stenosis \\
Hypoplastic left heart syndrome \\
Truncus arteriosus \\
Obstructed TAPVR \\
Complex Univentricular hearts with pulmonary atresia \\
Aortic stenosis \\
Coarctation of aorta \\
Interrupted aortic arch
\end{tabular}

Even in the developed resourceful countries with active screening programs, Chang et al. [4] reported 30 neonates dying each year of missed CCHD at birth in California. They found the median age of demise to be less than 2 weeks, concluding that these were preventable by careful cardiovascular examination for left sided obstructive lesions in the Pediatricians office in an early post natal visit at 3-5 days of life.

\section{CLUES TO DIAGNOSIS OF CONGENITAL HEART DISEASE}

Neonates with CHD have vague symptoms of poor feeding, lethargy or tachypnea. These symptoms are also seen in many other serious and perhaps more common neonatal diseases like sepsis, pneumonia, and metabolic disorders.

Children with CHD may have minimal or obvious symptoms. Lesions with excessive pulmonary blood flow present with tachypnea which becomes pronounced during milk feeding or exercise. Easy fatigability and excessive sweating are features of left to right shunt of significant magnitude. Recurrent respiratory infections may be a clue to CHD.

Peripheral cyanosis or acrocyanosis is more common and is a physiological response to certain conditions like exposure to cold temperatures, fright, or nervousness. This is due to vasoconstriction of skin blood vessels and should not be confused with central cyanosis. Central cyanosis due to CHD is because of arterial desaturation, and is present at all times, not intermittently. It is initially seen on the inner surface of lips and tongue. Naked eye may not appreciate mild cyanosis. Moderate degree of cyanosis may be noted on finger and toe nails.

Routine examination of newborn babies detects less than half of them with CHD because the majority of them are asymptomatic and signs may be subtle [12]. A normal examination does not rule out CHD. In fact severe CHD may not have any murmur. In particular, left heart obstructive lesions like coarctation of aorta and interrupted aortic arch can easily be overlooked. On the other hand, a normal newborn can have physiological murmur of a patent ductus arteriosus, pulmonary artery branch hypoplasia or tricuspid regurgitation. Therefore, only a high index of suspicion will pick up this group of diseases. Considering that at least half of the newborns with murmur may have a structural heart disease, they should be referred for echocardiogram [13]. Older children will have signs of abnormal peripheral pulses (high or low volume), hyperdynamic precordium, abnormal heart sounds and murmurs. Flaring of alai nasi, intercostal and subcostal chest retractions should alert a physician to an underlying CHD.

Hypercyanotic spell is a complication of cyanotic heart diseases with reduced pulmonary blood flow and is certainly not an early symptom. Similarly, headache, vomiting, fever, seizures and stroke indicate cerebral complications. Presence of a systemic disease or a syndrome should alert a physician to an associated cardiac anomaly. Midline defects may also have CHD [14].

Abnormal cardiac silhouette on a chest X-ray done for suspected lung disease is a clue to an underlying heart disease.

\section{SCREENING PROGRAMS}

Early detection of CCHD is defined as its diagnosis being made during birth hospitalization and late detection is diagnosis after being discharged from hospital [6]. In the developed countries, pulse oximetry screening (POS) is routinely done in all newborn babies before being discharged after birth. Critical congenital heart disease can be picked up by this simple test. Oxygen saturation by pulse oximetry can be measured at a post ductal location (distal to the insertion of patent ductus arteriosus which is mostly the lower limb) or combination of pre ductal (which is proximal to the insertion of patent ductus arteriosus, which is usually the upper $\operatorname{limb}$ ) and post ductal and the acceptable normal value is 95\% [15]. In the West, screening programs vary between centers. The American Academy of Pediatrics and the American Heart Association recommend screening newborns at 24 to 48 hours of life with use of both pre- and post-ductal measurements [16]. An oxygen saturation in the right hand and foot of $>95 \%$ makes the screening negative [13]. POS may not detect acyanotic heart diseases like ventricular septal defect, atrial septal defect or a patent ductus arteriosus. These are not considered 
critical defects. POS will detect cyanotic heart diseases and duct dependent acyanotic CCHD which may need urgent intervention.

In a study from Pakistan, the sensitivity, specificity, positive predictive value, and negative predictive value of pulse oximetry screening were $32 \%, 99.5 \%, 50 \%$, and $98.9 \%$ respectively [17]. In another study from the developed world, the accuracy of POS for CCHD had a sensitivity of around $43 \%$ and a specificity of $99.9 \%$.

Several studies have reported improved diagnostic value of a combined careful clinical examination and POS [18] with an improvement in sensitivity to 51\% [6]. Yet another study showed the sensitivity of the combined method of evaluation by clinical examination and POS to be $77 \%$, and for POS alone it was $31 \%$, and $46 \%$ for clinical examination alone [19].

Diagnostic accuracy of clinical examination alone is very subjective, based on geographical region and related to approach to diagnosing $\mathrm{CHD}$ in different training set-ups. In the developed countries, clinical findings are losing diagnostic value and more reliance is on investigations. However, in a developing country, clinical suspicion or diagnosis is still an important approach to patient management.

Echocardiogram is the gold standard for accurate diagnosis and patients with suspected CHD should be referred to a Pediatric Cardiologist for evaluation and echocardiography.

\section{DELAYED OR MISSED DIAGNOSIS AND ITS CONSEQUENCES}

Mortality of neonates diagnosed late with CCHD is double that of those diagnosed early [4]. Timely diagnosis will help in timely intervention, either catheter based or surgical, because major contributors to the outcome are clinical deterioration and collapse prior to diagnosis and treatment [13]. In California, the incidence of missed CCHD diagnosis was 1.7/100,000 live births and no statistical difference was found in the incidence among racial/ethnic groups [4]. In a study from UK, 30\% of $\mathrm{CCHD}$ were diagnosed late and $5 \%$ were diagnosed after death [13]. Delayed or missed diagnosis of CHD was found in $0.14 / 1000$ live births in a study from US. Hence, they calculated that $30-50 \%$ of CCHDs will be undiagnosed on discharge from hospital and $10 \%$ of them will die before timely diagnosis is made [13]. This diagnostic gap is probably much higher in the developing countries. There is paucity of data on this subject from Pakistan where delayed diagnosis, delayed referrals and adults presenting with CHD for the first time is quite common (unpublished observation).

\section{ANTENATAL (FETAL) ECHOCARDIOGRAPHY}

Early diagnosis definitely has improved outcome in terms of survival and morbidity [17]. Fetal echocardiography, done between 18-24 weeks of gestation [13] (ideally between 18-20 weeks), provides a number of options for fetuses diagnosed in utero with mild, moderate or severe types of CHD. Before 24 weeks of gestation, complex CHDs with poor post natal outcome can be given the option of discontinuation of the pregnancy. Alternatively, plans not to intervene after birth can be discussed. Parents are better prepared to handle such stressful situations if diagnosed antenatally.

For CHDs requiring post natal intervention, the advantages of antenatal diagnosis is planning delivery of the neonate at a center equipped to handle these emergencies as well as arranging finances for such interventions. Timely transferal of the mother before delivery will help prompt and management, thereby minimizing the morbidity from acidosis, low cardiac output, hypoxemia, delayed intubation and ventricular dysfunction, which are inevitable complications of delayed treatment.

Although this field of antenatal diagnosis continues to evolve in Pakistan and many other countries, fetal echocardiography can diagnose from $8-48 \%$ of $\mathrm{CHDs}$ depending on centers expertise. It was seen that in a University based practice in the US [13], detection rates were higher than in a non-university based practice (71$100 \%$ compared to $0-39 \%$ respectively).

\section{CONCLUSION}

There is abundant information to support that early detection of CHD improves outcome. Early diagnosis is ideally made at birth, before hospital discharge, so that the family is counseled and management plans are discussed. Critical congenital heart disease is a medical emergency in newborns, infants as well as children. Early diagnosis of non-critical CHDs should be made within the first few weeks or months. Delay in diagnosis will lead to progress in the natural history of the disease; majority of them will either become a high risk for surgery or may be deemed inoperable. Therefore, timely referral to a center equipped to handle neonates, infants and children for appropriate intervention, will reduce morbidity and mortality.

\section{RECOMMENDATIONS}

There is a definite need to emphasize on approach to early diagnosis of CHD. First, all high risk pregnancies or those with risk of $\mathrm{CHD}$ should have a fetal echocardiogram between 18-24 weeks of gestation, ideally between 18-20 weeks, and a follow up management planned in cases of antenatally diagnosed CHDs. Secondly, all neonates must have a proper clinical examination before discharge (average 3 days after birth) in which cardiac murmur and adequacy of lower limb pulses are carefully looked for. Pulse oximetry screening, preferably both pre and post ductal, should be made mandatory in all well baby nurseries. Post natal visit to the Pediatrician or Family physician should be no later than 5 days of life 
so that life threatening CCHDs are not missed. Routine pulse oximetry and careful examination of pulses, abnormal heart sounds and murmur in infants and older children should alert heath care providers of underlying $\mathrm{CHD}$ warranting further investigations.

\section{CONFLICT OF INTEREST}

The author declares no conflict of interest.

\section{ACKNOWLEDGEMENTS}

\section{Declared none.}

\section{REFERENCES}

1. Zeng Z, Zhang H, Liu F, Zhang $\mathrm{N}$. Current diagnosis and treatments for critical congenital heart defects (review). Exp Therap Med 2016; 11: 1550-4.

2. Rizvi SF, Mustafa G, Kundi A, Khan MA. Prevalence of congenital heart disease in rural communities of Pakistan. J Ayub Med Coll 2015; 27(1): 124-7.

3. Hassan I, Haleem AA, Bhutta ZA. Profile and risk factors for congenital heart disease. J Pak Med Assoc 1997; 47(3): 78-81.

4. Chang R-KR, Gurvitz M, Rodriguez S. Missed diagnosis of critical congenital heart disease. Arch Pediatr Adolesc Med 2008; 162(10): 969-74.

5. Pakistan demographic profiles 2019 Index Mundi. Available from: www.indexmundi.com/pakistan/demographics_profile (Accessed on April 13, 2020).

6. Piazza L, Micheletti P, Negura D, Arcidiacono C, Saracino A Carminati M. Early diagnosis of congenital heart disease: when and how to treat? In: Neonatology: practical approach to neonatal diseases; Buonocore G, Bracci R, Weindling M, Eds. Berlin: Springer Science \& Business Media 2012: pp. 569-76.

7. Haq FU, Jalil F, Hashmi SK, Jumani MI, Imdad A, Jabeen M, et al. Risk factors predisposing to congenital heart defects. Ann Pediatr Card 2011; 4: 117-21.
8. Yunis K, Mumtaz G, Bitar F, Chamseddine F, Kassar M, Rashkidi $\mathrm{J}$, et al. Consanguineous marriage and congenital heart defects: a case-control study in the neonatal period. Am J Med Genet A 2006; 140(14): 1524-30

9. Bittles $\mathrm{AH}$. Assessing influence of consanguinity on congenital heart disease. Ann Pediatr Cardiol 2011; 4(2): 111-6.

10. Nabulsi MM, Tamim H, Sabbagh M, Obeid MY, Yunis KA, Bitar FF. Parental consanguinity and congenital heart malformations in a developing country. Am J Med Genetics 2003; 116A: 342-7.

11. Liske MR, Greeley CS, Law DJ, Reich JD, Morrow WR, Baldwin HS, et al. Report of the Tennessee Task Force on screening newborn infants for critical congenital heart disease. Pediatrics 2006; 118: 1250-6.

12. Richmond S, Wren $\mathrm{C}$. Early diagnosis of congenital heart disease. Semin Neonatol 2001; 6(1): 27-35.

13. Kumar P. Universal pulse oximetry screening for early detection of critical congenital heart disease. Clin Med Insights Pediatr 2016; 10: $35-41$.

14. Shafi T, Khan MR, Atiq M. Congenital heart disease and associated malformations in children with cleft lip and palate in Pakistan. $\mathrm{Br} \mathrm{J}$ Plastic Surg 2003; 56: 106-9.

15. Danworapong S, Nakwan N, Napapongsuriya C, Choksuchat D, Danworaphong S. Assessing the use of pulse oximetry screening for critical congenital heart disease in asymptomatic term newborns. J Clin Neonatol 2019; 8: 28-33.

16. Ailes EC, Gilboa SM, Honein MA, Oster ME. Estimated number of infants detected and missed by critical congenital heart defect screening. Pediatrics 2015; 135(6): 1000-8.

17. Mohsin M, Humayun KN, Atiq M. Clinical screening for congenital heart disease in newborns at a tertiary care hospital of a developing country. Cureus 2019; 11(6): e4808.

18. Brunetti ND, Rosania S, D'Antuono C, et al. Diagnostic accuracy of heart murmur in newborns with suspected congenital heart disease. J Cardiovasc Med 2015; 16(8): 556-61.

19.Bakr A, Habib, H. Combining pulse oximetry and clinical examination in screening for congenital heart disease. Pediatr Cardiol 2005; 26: 832-5. 